C.M. Smith

\title{
Evolutionary and archaeological perspectives on estimating the likelihood of civilization collapse
}

\begin{abstract}
The likelihood that modern civilization will collapse has been estimated many times for many reasons. From evolutionary and archaeological perspectives, the repeated disintegration of ancient civilizations (none have survived more than a few millennia) is unsurprising. Can we expect modern civilization to also collapse? This is likely in the absence of proactive, self-preserving methods, which may be informed by survival strategies known to evolutionary biology. Such methods include deployment of technologies to realize the current potential of modern civilization (and the modern genomes we select to protect) to be the Earth's longest-lived complex adaptive system. I suggest some of these methods, which include the use of technology to spread humanity from Earth to other solar system habitats.
\end{abstract}

Nanotechnology Perceptions 13 (2017) 116-122

doi: 10.4024/N09SM17A.ntp.13.02 